Published in final edited form as:

Crit Care Clin. 2012 January ; 28(1): 25-v. doi:10.1016/j.ccc.2011.09.004.

\title{
Economics of ICU Organization and Management
}

\author{
Hannah Wunsch, MD, MSc ${ }^{1,2}$, Hayley Gershengorn, MD $^{3}$, and Damon C. Scales, MD, PhD ${ }^{4,5}$ \\ ${ }^{1}$ Department of Anesthesiology, Columbia University, New York, NY, USA \\ ${ }^{2}$ Department of Epidemiology, Columbia University, New York, NY, USA \\ ${ }^{3}$ Division of Pulmonary, Critical Care, and Sleep Medicine, Beth Israel Medical Center, New York, \\ NY, USA \\ ${ }^{4}$ Interdepartmental Division of Critical Care Medicine, University of Toronto, Toronto, Canada \\ ${ }^{5}$ Department of Critical Care Medicine, Sunnybrook Health Sciences Centre, Toronto, Canada
}

\section{Keywords}

Critical Care; Intensive Care Unit; Length of Stay; Staffing; Organization; Economics

\section{INTRODUCTION}

\begin{abstract}
Intensive care is an integral but expensive component of healthcare in developed countries [1]. An estimate in the US is that fully $2 \%$ of the population receives intensive care every year [2], and overall the percentage of patients who receive intensive care before they die is increasing [3,4]. Projections of the need for mechanical ventilation predict an exponential growth in the coming years due to the aging population and their over-representation among mechanically ventilated cohorts [5]; this increase in need for mechanical ventilation will be associated with increasing costs of intensive care [6,7]. Much of the focus of intensive care is on improvements in technology for organ support and resuscitation. Yet quality healthcare also involves appropriate organization of resources, with the potential to both impact patient outcomes and the costs of the care provided. These economic considerations are likely to become increasingly important as the demand for critical care increases in the face of limited resources.
\end{abstract}

The economics of organizing the delivery of intensive care can focus on the management of human resources and operating costs within an ICU itself, or the use of ICU resources within a healthcare system. This article will focus on both these perspectives, emphasizing issues related to optimal staffing and the economic consequences of different staffing choices.

Perspective regarding costs-The costs of providing critical care can be considered in short-term and long-term time horizons and are incurred to varying degrees by patients,

\footnotetext{
Address for correspondence: Hannah Wunsch, MD, MSc, Department of Anesthesiology, Columbia University, 622 West $168^{\text {th }}$ St, PH5-527D, New York, NY 10032, USA, Tel: +1 (212) 305-0679, Fax: +1 (212) 305-8287, hw2125@ columbia.edu.

Hayley Gershengorn, MD, Division of Pulmonary, Critical Care, and Sleep Medicine, Beth Israel Medical Center, First Avenue at $16^{\text {th }}$ Street, 7 Dazian, New York, NY 10003

Damon C. Scales, MD PhD, Department of Critical Care Medicine, Sunnybrook Health Sciences Centre, Interdepartmental Division of Critical Care, University of Toronto, 2075 Bayview Avenue, Room D108, Toronto, Ontario, Canada, M4N 3M5, 416-480-6100 ext 83734, damon.scales@utoronto.ca
}

Disclosures: The authors have nothing to disclose 
hospitals, insurance companies, the government or other payer, and society as a whole. Thus, the first question to ask when evaluating the economic impact of any organizational change affecting the ICU is which party actually accrues a cost change [8]. In this article, we will primarily focus on the potential economic implications of organization and management choices from the perspective of the individual hospital.

Fixed versus variable costs in the ICU-Hospital costs are comprised of fixed and variable costs. In brief, the fixed costs remain constant and are independent of small changes in the number of patients being cared for in the hospital. They also generally reflect the operational costs required to provide care [9]. Examples of fixed costs in the ICU include staff salaries, the money paid to purchase mechanical ventilators, and the maintenance required on the building. Variable costs are the hospital costs associated with the care of individual patients, and will fluctuate with patient volumes. Examples of variable costs are the costs of specific medications the patient receives or the cost of an additional central venous catheter inserted [10]. The majority of costs associated with care in the hospital are fixed costs, often estimated to account for over $80 \%$ of total costs [9]. Whether or not hospital (or ICU) beds are occupied, the hospital continues to pay the fixed costs of care, and therefore most cost reductions associated with any system change will be small if due to changes in variable costs only.

The economics of intensive care from the perspective of the hospital also depend on how a hospital is reimbursed by a health system [8]. For example, under one type of payment system, a hospital receives a set amount of money for the care of all patients, regardless of the number that are actually admitted. Another option is a fixed level of reimbursement to the hospital to provide care for each patient admitted with a specific diagnosis or surgical procedure. In these situations, the actual components of treatment that are provided to an individual patient are not reimbursed separately, but instead the hospital receives a lump sum based upon expected costs. In a "per diem" model, the hospital is paid an additional sum for each day that a patient remains hospitalized. Hospitals can also be paid using a feefor-service model, receiving a sum of money for each additional test, procedure, medication, etc. that is provided to each patient. Within each of these payment schemes, there are opportunities for the hospital to change the system to maximize revenue. In this article, we primarily consider the actual costs of providing treatment when discussing strategies for reducing total costs, rather than strategies to improve the economic outlook for the hospital based on different payment schemes.

\section{Costs within the ICU}

Decreasing Length of Stay-Many studies in critical care target reductions in ICU length of stay and equate this outcome with a "cost savings". In reality, large cost savings will only be realized if the reductions in ICU length of stay result in a reduced number of total admissions and consequent reductions in number of ICU beds and fixed costs of care [11]. In addition, one must cognizant of the concept of "cost-shifting", in which reductions in costs in one area are accompanied by rises in costs elsewhere in order to address clinical needs. In most situations the actual cost savings associated with decreased ICU length of stay therefore comprise only a small fraction of total costs. For example, Kahn and colleagues analyzed the potential cost savings attributable to reductions in ICU length of stay for ICU survivors who had received mechanical ventilation and ICU admission of more than three days. The authors found that the mean variable costs of the last day in the ICU was $\$ 397$, while the cost of the next day on the hospital ward was $\$ 279$; thus, reducing ICU length of stay by one day would only result in a cost savings of $0.2 \%$ of all hospital expenditure [12]. Conversely, if there is typically high demand for ICU resources (and the absolute number of patients in the ICU remains relatively constant), reducing ICU length of 
stay can paradoxically increase variable costs because higher acuity patients requiring more intensive and expensive treatments replace the lower-acuity patients who are discharged [12]. Moreover, the overall economic effect of accommodating an additional ICU patient may be different depending on the type of ICU and the type of patient. For example, if any decrease in one patient's ICU length of stay helps avoid the cancellation of an elective surgery such as coronary artery bypass grafting for another patient, the actual economic impact on the hospital may be different than providing admission for an additional patient with pneumonia, but will also depend on how the hospital is reimbursed, as described earlier. In this article we address specific organizational aspects of the ICU by focusing on the actual costs incurred by providing direct care and, where possible, avoid inferences based on economic implications of reducing ICU length of stay. However, we generally consider interventions that decrease ICU length of stay to be desirable.

Staffing-The largest and primarily fixed costs of operating an ICU are staff salaries, which are estimated to account for over $50 \%$ of fixed costs for all hospitalized patients in the US [9] and 33\% to $69 \%$ of total ICU costs in other countries (see Figure 1) [13-20]. Staffing patterns in the ICU vary markedly between ICUs and between health systems, with variations in the specialty of the physicians, nurse to patient ratios, and the presence of other healthcare professionals on the team. Yet, the large proportion of costs in the ICU taken up by the compensation for its staffseems to be relatively consistent across many countries, despite the different staffing patterns and healthcare systems.

Nursing-Nurses are an integral, if not the most vital, component of all ICU teams, but perhaps paradoxically, nurse-to-patient staffing ratios and other issues related to the provision of ICU nursing care differ from country to country, from region to region, and even may differ between ICUs in a particular hospital. One European study, for example, from the 1990s found that the majority of ICU nurses cared for two patients, but also observed substantial variation between the planned nurse to patient ratios and actual staffing ratios in the ICU [21]. Studies conducted outside of the ICU have shown that higher nurse to patient ratios and more highly trained nursing staff are associated with fewer adverse events [22] and possibly decreased hospital mortality [23]. Fewer data are available to inform the most cost effective nurse-to-patient ratio in an ICU, although some studies have suggested that higher nursing ratios decrease adverse event rates and lead to better patient outcomes $[22,24,25]$. Obviously, maintaining a higher nurse to patient ratio increases the fixed costs of intensive care and is a major barrier to providing one-on-one nursing in most ICUs (Table 1). It is also possible that savings assocaited with reductions in adverse events for patients could offset the higher fixed costs with higher nursing ratios, but this possibility remains speculative. Some ICUs have adopted a "flex" system that flexibly schedules nurses based on the anticipated workload in the unit at that time, rather than staffing based on the number of admitted patients. This structure may be financially beneficial for the hospital and may also improve nursing satisfaction by matching staff to workload demands [26]. However, this model may also result in unpredictable working hours and salaries for individual nurses

Intensivist Staffing-A great deal of attention is focused on the role of intensivists in the management of critically ill patients, particularly in the United States where there is a mix of staffing systems with only approximately one-third of ICUs covered by intensivists [27]. Overall, most studies demonstrate that intensivist staffing in the ICU improves clinical outcomes [28] and both the American College of Critical Care and the Society of Critical Care Medicine recommend intensivist coverage [29]. However, these studies have varied in the definition of a "closed" or "open" ICU and the number of hours of intensivist coverage, and are hampered by potential selection biases associated with which patients received intensivist coverage as well as other factors such as the type of ICU, concomitant staffing 
patterns, and ICU culture [30,31]. Few studies have considered the cost-effectiveness of intensivist staffing. One simulation of intensivist implementation demonstrated cost savings (for the hospital), depending on the size of the ICU, but involved many large assumptions to draw conclusions regarding the impact of intensivists on the hospital system as a whole [32].

Recent discussions have questioned whether 24 hour in-house intensivist coverage might lead to additional improvements in patient outcomes compared to daytime-only intensivist coverage [33]. One study demonstrated improved compliance with recommended processes of care, but no effect on hospital mortality with 24 hour in-house coverage [34]. Recent work by Banerjee et al examined 24-hour in-house intensivist coverage (versus daytime only) and demonstrated a decreased length of stay for the sickest patients admitted at night and cost savings associated with the decreased length of stay [35]. The study did include estimates of the costs of additional intensivists, but it did not clearly differentiate between fixed and marginal costs of care. Thus it may have potentially over-estimated the costsavings associated with the decreased length of stay.

Multidisciplinary teams-Non-physician team members have a large role in the ICU $[36,37]$. Data suggest that multidisciplinary teams on rounds can potentially impact the mortality [38] and length of stay of patients in the ICU [39]. However, expanding the membership of the multidisciplinary team, especially non-nursing healthcare workers, may also increase fixed costs in an ICU (Table 1).

Pharmacists have become an integral component of many ICU teams and several studies demonstrate the economic impact of clinical pharmacists in the ICU. One study in particular detailed the changes made in medication management with the input of a clinical pharmacist over a three month period, with a substantial portion of the consultations $(47.1 \%)$ resulting in decreased drug costs [40]. More recent studies have examined the impact of clinical pharmacists on management of particular groups of patients, such as critically ill patients with thrombo-embolic or infarction related events [41] and infections [42]. Both studies demonstrated that direct involvement of pharmacists in care led to decreased charges for medications. However, it is important to note that the decreased variable costs may be offset by the fixed costs of the additional salaries.

Perhaps the more important benefit of pharmacists is the potential to decrease adverse drug events [43]. Preventable adverse drug events in the ICU may occur twice as frequently as on the regular hospital ward, primarily due to the greater number of drugs ordered in the ICU [44], thus making the ICU a prime target for improvement in this area. One study demonstrated a decrease in prescribing errors by two-thirds with the addition of a senior pharmacist on rounds in the ICU [45]. The cost-effectiveness of pharmacists should therefore consider not only the pharmacist's salary and prescribing costs, but also the potential reduction in the incidence of expensive complications.

Respiratory therapists are common in North American ICUs and often assume important clinical roles, especially with respect to ventilator management. The use of respiratory therapists varies in other countries. Outside of the ICU respiratory therapist-initiated treatment protocols have led to better compliance with institutional algorithms for care $[46,47]$. In the ICU, respiratory therapists have been shown to improve compliance with weaning protocols and decreased duration of mechanical ventilation $[48,49]$. Guidelines for Weaning and Discontinuing Ventilatory Support recommend that protocols designed for nonphysician health-care professionals should be developed and implemented by ICUs [50]. However, the impact of respiratory therapists on both patient mortality [51] and the economics of care is still not well defined. 
The role of the physical therapist in the ICU appears to be evolving to include early rehabilitation, including mobilization of mechanically ventilated patients. Several recent studies have suggested that early rehabilitation may leads to improved patient outcomes, including functional status [52,53] and length of stay [52-54]. The full economic impact along with the cost-effectiveness of this intervention requires further study, but limited evidence suggests that this therapy may not lead to increase costs of care, even after accounting for the salaries of the physical therapy team [52]. Further research to evaluate the impact of this intervention is required, but the potential for large system-wide savings may also exist if some of these patients no longer require additional care in nursing or rehabilitation facilities due to early intervention.

Finally, the role of a palliative care team in the ICU, either as a separate consult team or as part of the ICU team itself is still being defined, and the potential financial implications are not yet well explored. No studies have specifically addressed the financial impact of palliative care in patients in the ICU, but introducing these teams may lead to less use of intensive care (in subsets of patients) [55] and reductions in ICU length of stay [56,57]. However, the cost savings of these interventions may be limited since these patients represent a relatively small proportion of patients who (may) be cared for in the ICU and are likely to influence only the marginal costs of care [11]. It remains unknown whether reducing use of ICU at the end of life through aggressive palliative care can lead to any substantial impact on costs of care.

Standardization of care-Health technology in the ICU, such as mechanical ventilators, pulmonary artery catheters, and other monitoring devices, may represent either fixed or variable costs. Many of these ICU technologies have a limited evidence base supporting their use, and could be considered targets for cost-reduction strategies. The use of intensive care technology has been shown to vary widely among different intensivists working in the same ICU, with no discernible variation in patient outcomes. In one study, the daily discretionary costs of care varied by $43 \%$ across different intensivists, with a mean difference of $\$ 1,003$ per admission and no differences in ICU length of stay or hospital mortality [58]. Reducing use of technology and equipment that have not been linked to improved patient outcomes will likely decrease costs [59], but can be slow to occur if clinicians consider these to be an integral part of ICU care. A recent example of changing practice is the use of pulmonary artery catheters; after multiple studies failed to demonstrate any clinical benefit associated with their systematic use in different ICU populations, the frequency of insertion has dropped dramatically in the United States [60,61].

Standardization of treatment approaches and the use of protocols to help organize ICU care can help reduce the use of unproven and expensive treatments (or at least ensure that they are used only in situations that are supported by strong levels of evidence) and also may lead to increased use of evidence-based therapies and improved patient outcomes. There are many ways to approach standardization of care which include the addition of multidisciplinary staff (as described above), the implementation of checklists [62,63], prompting and the use of clinical reminders [63], and the adoption of clinical protocols and treatment "bundles" [64]. Some of these options have been examined as individual components (such as checklists [65]) and others as "bundles" of care to be delivered together $[64,66]$. The combination of checklists on rounds with a "prompter" to ensure that the elements of the checklist were addressed was associated in one single-center study with a decreases in mortality and length of ICU stay, potentially decreasing variable costs associated with care [63]. However, the fixed costs of requiring additional staff to act as "prompter" may offset the potential economic benefit of this intervention (see Table 1). 
Many ICUs and hospitals have implemented protocols to limit the use of expensive technologies and treatments to their appropriate and evidence based indications as a strategy to reduce costs and "indication creep". For example, there has been a substantial increase in off-label use of Recombinant Factor VIIa [67], with little evidence to support its administration in many cases [68]. In the ICU, even a test as basic as an arterial blood gas may be subject to overuse, with one study demonstrating a substantial decrease in the number of arterial blood gas requests with implementation of guidelines and feedback [69].

\section{Costs within the healthcare system}

Organization of admission and discharge practices and alternatives to careSince ICU care is almost always more expensive than the care provided on a general ward [12], choosing to not admit a patient to the ICU will likely decrease the costs of care for that individual. However, such decisions will also likely lead to worse outcomes if appropriate and potentially life-saving treatments are withheld; decreasing the use of intensive care is therefore only a feasible approach to decreasing costs if the admission to the ICU is not appropriate. One study examined the factors associated with being a "high performance" ICU (defined as having a standardized mortality ratio of 1.0 or less) and found that these high performing units all had ICU directors (or a designee) who were authorized to refuse admission to patients not meeting appropriate criteria and to triage requested admissions to extended-stay recovery rooms and intermediate care areas [70]. Cost savings for the hospital may also be realized if sub-acutely or chronically critically ill patients are discharged more expeditiously from the ICU. Although as noted earlier, decreasing ICU length of stay by small amounts (such as a single day) may do little to impact costs of care [12]. However, patients with ongoing respiratory failure traditionally have had few options for care once their needs for acute intensive care are over, yet often stay in an ICU for extended periods. Different institutions attempt to accommodate these patients outside the traditional ICU setting in different manners. The designation of a flexibly sized section of the surgical ICU for the "subacutely ill" allowed for reductions in costly resources (e.g., nursing) without the additional cost of building a separate step-down facility [71]. Similarly, several studies demonstrate that the creation of a physically separate step-down unit may result in reduced costs of care [72-75]. Another option in some hospitals, particularly in the United States, is to transfer patients quickly out of the acute hospital to receive prolonged care elsewhere. The use of long-term acute care facilities (which can care for mechanically ventilated patients) in the United States has increased dramatically over the past decade [76]. Whether the movement of patients to these facilities is cost effective for the healthcare system as a whole is unclear, but there may be a substantial decrease in costs for the acute care hospital if patients are discharged much earlier.

Regionalization-A broader approach to triage of patients to the most appropriate setting is regionalization of ICU beds and care of ICU patients, particularly mechanically ventilated patients. In the United States this idea has been proposed based on data suggesting that outcomes may be improved for mechanically ventilated patients cared for at higher volume hospitals [77]. Regionalized systems exist for both trauma and neonatal care [78,79], and some regionalization occurs in most countries, either through formal systems [80], or informal networks [81]. However, the barriers to complete regionalization of intensive care are substantial, including concerns regarding strain on patients' families, lack of strong central authority to organize triage, and the potential to overwhelm capacity at larger hospitals [82]. The impact of regionalization of intensive care for the economics of hospitals is also uncertain, with concern that smaller hospitals may be hurt financially, while larger hospitals receiving patients may not have enough resources [83]. 
Alternatively, telemedicine could allow for an increased reach of critical care expertise in remote ICUs by providing access to intensivists. These physicians may offer either monitoring or consultation as needed, and theoretically provide the associated benefits for patient care seen in studies of intensivist staffing [28]. However, the results are inconsistent [84]; two multi-center studies were unable to demonstrate an association between the use of telemedicine and patient outcomes $[85,86]$, while one has shown improvements for patients [87], and a fourth demonstrated some economic benefit [88].

Assessing the potential impact of telemedicine programs is hampered by the fact that their adoption has often been studied in ICUs that already have high staffing ratios. The true benefit may be found only in small hospitals with limited access to intensivist care. Telemedicine could also be used as a tool to improve the implementation of specific interventions, and to facilitate adherence to current best practice, such as lung protective ventilation or early-goal directed therapy [89].

Conclusion-The ICU is a complex system and the economic implications of altering care patterns in the ICU can be difficult to unravel. While the clinical impact of many aspects of organization and management have been studied in the ICU, few studies have specifically examined the economics of implementing organizational and management changes. Even fewer have acknowledged the many competing economic interests of patient, hospital, payer and society. It does appear, however, that for certain aspects of ICU organization (e.g., the inclusion of a staff pharmacist on a multidisciplinary ICU team) there may be an alignment of clinical and financial goals for all parties. With continuously increasing healthcare costs there is a great need for more studies focused on economics to inform the optimal organization of the ICU. Ideally these studies should not focus solely on reductions in ICU length of stay, but should strive to measure the true costs of care within a given healthcare system.

\section{Acknowledgments}

Funding: Award Number K08AG038477 from the National Institute On Aging to Hannah Wunsch

\section{Reference List}

1. Wunsch H, Angus DC, Harrison DA, et al. Variation in critical care services across North America and Western Europe. Crit Care Med. 2008; 36(10):2787-2789. [PubMed: 18766102]

2. Kersten A, Milbrandt EB, Rahim MT, et al. How big is Critical Care in the U.S.? [Abstract]. Crit Care Med. 2003; 31(Suppl):A8.

3. Barnato AE, McClellan MB, Kagay CR, Garber AM. Trends in inpatient treatment intensity among Medicare beneficiaries at the end of life. Health Serv Res. 2004; 39(2):363-375. [PubMed: 15032959]

4. Angus DC, Barnato AE, Linde-Zwirble WT, et al. Use of intensive care at the end of life in the United States: an epidemiologic study. Crit Care Med. 2004; 32(3):638-643. [PubMed: 15090940]

5. Carson SS, Cox CE, Holmes GM, Howard A, Carey TS. The changing epidemiology of mechanical ventilation: a population-based study. J Intensive Care Med. 2006; 21(3):173-182. [PubMed: 16672639]

6. Fisher ES, Bynum JP, Skinner JS. Slowing the growth of health care costs--lessons from regional variation. N Engl J Med. 2009; 360(9):849-852. [PubMed: 19246356]

7. Sutherland JM, Fisher ES, Skinner JS. Getting past denial--the high cost of health care in the United States. N Engl J Med. 2009; 361(13):1227-1230. [PubMed: 19741220]

8. Kahn JM. Understanding economic outcomes in critical care. Curr Opin Crit Care. 2006; 12(5):399_ 404. [PubMed: 16943716] 
9. Roberts RR, Frutos PW, Ciavarella GG, et al. Distribution of variable vs fixed costs of hospital care. JAMA. 1999; 281(7):644-649. [PubMed: 10029127]

10. Rossi C, Simini B, Brazzi L, et al. Variable costs of ICU patients: a multicenter prospective study. Intensive Care Med. 2006; 32(4):545-552. [PubMed: 16501946]

11. Luce JM, Rubenfeld GD. Can health care costs be reduced by limiting intensive care at the end of life? Am J Respir Crit Care Med. 2002; 165(6):750-754. [PubMed: 11897638]

12. Kahn JM, Rubenfeld GD, Rohrbach J, Fuchs BD. Cost savings attributable to reductions in intensive care unit length of stay for mechanically ventilated patients. Med Care. 2008; 46(12): 1226-1233. [PubMed: 19300312]

13. Noseworthy TW, Konopad E, Shustack A, Johnston R, Grace M. Cost accounting of adult intensive care: methods and human and capital inputs. Crit Care Med. 1996; 24(7):1168-1172. [PubMed: 8674330]

14. Dickie H, Vedio A, Dundas R, Treacher DF, Leach RM. Relationship between TISS and ICU cost. Intensive care medicine. 1998; 24(10):1009-1017. [PubMed: 9840233]

15. Rechner IJ, Lipman J. The costs of caring for patients in a tertiary referral Australian Intensive Care Unit. Anaesthesia and intensive care. 2005; 33(4):477. [PubMed: 16119489]

16. Moran JL, Peisach AR, Solomon PJ, Martin J. Cost calculation and prediction in adult intensive care: a ground-up utilization study. Anaesthesia and intensive care. 2004; 32(6):787-797. [PubMed: 15648989]

17. Tan SS, Hakkaart-van Roijen L, Al MJ, et al. Review of A Large Clinical Series: A Microcosting Study of Intensive Care Unit Stay in the Netherlands. Journal of intensive care medicine. 2008; 23(4):250-257. [PubMed: 18508839]

18. Graf J, Graf C, Janssens U. Analysis of resource use and cost-generating factors in a German medical intensive care unit employing the Therapeutic Intervention Scoring System (TISS-28). Intensive care medicine. 2002; 28(3):324-331. [PubMed: 11904663]

19. Moerer O, Plock E, Mgbor U, et al. A German national prevalence study on the cost of intensive care: an evaluation from 51 intensive care units. Critical care (London, England). 2007; 11(3):R69.

20. Martin J, Neurohr C, Bauer M, Weiss M, Schleppers A. [Cost of intensive care in a German hospital: cost-unit accounting based on the InEK matrix]. Anaesthesist. 2008; 57(5):505-512. [PubMed: 18389191]

21. Moreno R, Reis MD. Nursing staff in intensive care in Europe: the mismatch between planning and practice. Chest. 1998; 113(3):752-758. [PubMed: 9515853]

22. Heinz D. Hospital nurse staffing and patient outcomes: A review of current literature. Dimensions of critical care nursing. 2004; 23(1):44. [PubMed: 14734900]

23. Needleman J, Buerhaus P, Pankratz VS, Leibson CL, Stevens SR, Harris M. Nurse staffing and inpatient hospital mortality. N Engl J Med. 2011; 364(11):1037-1045. [PubMed: 21410372]

24. Amaravadi RK, Dimick JB, Pronovost PJ, Lipsett PA. ICU nurse-to-patient ratio is associated with complications and resource use after esophagectomy. Intensive Care Med. 2000; 26(12):18571862. [PubMed: 11271096]

25. Binnekade JM, Vroom MB, de Mol BA, de Haan RJ. The quality of intensive care nursing before, during, and after the introduction of nurses without ICU-training. Heart \& lung. 2003; 32(3):190196. [PubMed: 12827104]

26. Cho SH, June KJ, Kim YM, et al. Nurse staffing, quality of nursing care and nurse job outcomes in intensive care units. Journal of clinical nursing. 2009; 18(12):1729-1737. [PubMed: 19646118]

27. Angus DC, Shorr AF, White A, Dremsizov TT, Schmitz RJ, Kelley MA. Critical care delivery in the United States: distribution of services and compliance with Leapfrog recommendations. Crit Care Med. 2006; 34(4):1016-1024. [PubMed: 16505703]

28. Pronovost PJ, Angus DC, Dorman T, Robinson KA, Dremsizov TT, Young TL. Physician staffing patterns and clinical outcomes in critically ill patients: a systematic review. JAMA. 2002; 288(17): 2151-2162. [PubMed: 12413375]

29. Haupt MT, Bekes CE, Brilli RJ, et al. Guidelines on critical care services and personnel: Recommendations based on a system of categorization of three levels of care*. Critical Care Medicine. 2003; 31(11):2677-2683. [PubMed: 14605541] 
30. Levy MM, Rapoport J, Lemeshow S, Chalfin DB, Phillips G, Danis M. Association between critical care physician management and patient mortality in the intensive care unit. Ann Intern Med. 2008; 148(11):801-809. [PubMed: 18519926]

31. Pronovost PJ, Jenckes MW, Dorman T, et al. Organizational characteristics of intensive care units related to outcomes of abdominal aortic surgery. JAMA. 1999; 281(14):1310-1317. [PubMed: 10208147]

32. Pronovost PJ, Needham DM, Waters $\mathrm{H}$, et al. Intensive care unit physician staffing: financial modeling of the Leapfrog standard. Crit Care Med. 2006; 34(3 Suppl):S18-S24. [PubMed: 16477199]

33. Sapirstein A, Needham D, Pronovost PJ. 24-hour intensivist staffing: Balancing benefits and costs*. Critical Care Medicine. 2008; 36(1):367-368. [PubMed: 18158464]

34. Gajic O, Afessa B, Hanson AC, et al. Effect of 24-hour mandatory versus on-demand critical care specialist presence on quality of care and family and provider satisfaction in the intensive care unit of a teaching hospital*. Critical Care Medicine. 2008; 36(1):36-44. [PubMed: 18007270]

35. Banerjee R, Naessens JM, Seferian EG, et al. Economic implications of nighttime attending intensivist coverage in a medical intensive care unit*. Critical Care Medicine. 2011; 39(6):12571262. [PubMed: 21317642]

36. Curtis JR, Cook DJ, Wall RJ, et al. Intensive care unit quality improvement: A "how-to" guide for the interdisciplinary team*. Critical Care Medicine. 2006; 34(1):211-218. [PubMed: 16374176]

37. Nguyen YL, Wunsch H, Angus DC. Critical care: the impact of organization and management on outcomes. Current opinion in critical care. 2010; 16(5):487-492. [PubMed: 20689418]

38. Kim MM, Barnato AE, Angus DC, Fleisher LA, Kahn JM. The effect of multidisciplinary care teams on intensive care unit mortality. Arch Intern Med. 2010; 170(4):369-376. [PubMed: 20177041]

39. Smyrnios NA, Connolly A, Wilson MM, et al. Effects of a multifaceted, multidisciplinary, hospital-wide quality improvement program on weaning from mechanical ventilation. Crit Care Med. 2002; 30(6):1224-1230. [PubMed: 12072672]

40. Montazeri M, Cook DJ. Impact of a clinical pharmacist in a multidisciplinary intensive care unit. Crit Care Med. 1994; 22(6):1044-1048. [PubMed: 8205814]

41. MacLaren R, Bond CA. Effects of pharmacist participation in intensive care units on clinical and economic outcomes of critically ill patients with thromboembolic or infarction-related events. Pharmacotherapy. 2009; 29(7):761-768. [PubMed: 19558249]

42. MacLaren R, Bond CA, Martin SJ, Fike D. Clinical and economic outcomes of involving pharmacists in the direct care of critically ill patients with infections. Crit Care Med. 2008; 36(12): 3184-3189. [PubMed: 18936700]

43. Horn E, Jacobi J. The critical care clinical pharmacist: evolution of an essential team member. Crit Care Med. 2006; 34(3 Suppl):S46-S51. [PubMed: 16477202]

44. Cullen DJ, Sweitzer BJ, Bates DW, Burdick E, Edmondson A, Leape LL. Preventable adverse drug events in hospitalized patients: a comparative study of intensive care and general care units. Crit Care Med. 1997; 25(8):1289-1297. [PubMed: 9267940]

45. Leape LL, Cullen DJ, Clapp MD, et al. Pharmacist participation on physician rounds and adverse drug events in the intensive care unit. JAMA. 1999; 282(3):267-270. [PubMed: 10422996]

46. Kollef MH, Shapiro SD, Clinkscale D, et al. The effect of respiratory therapist-initiated treatment protocols on patient outcomes and resource utilization. Chest. 2000; 117(2):467-475. [PubMed: 10669692]

47. Stoller J, Mascha E, Kester L, Haney D. Randomized Controlled Trial of Physician-directed versus Respiratory Therapy Consult Service-directed Respiratory Care to Adult Non-ICU Inpatients. Am J Respir Crit Care Med. 1998; 158(4):1068-1075. [PubMed: 9769262]

48. Ely E, Bennett P, Bowton D, Murphy S, Florance A, Haponik E. Large Scale Implementation of a Respiratory Therapist-driven Protocol for Ventilator Weaning. Am J Respir Crit Care Med. 1999; 159(2):439-446. [PubMed: 9927355]

49. Marelich GP, Murin S, Battistella F, Inciardi J, Vierra T, Roby M. Protocol Weaning of Mechanical Ventilation in Medical and Surgical Patients by Respiratory Care Practitioners and Nurses*. Chest. 2000; 118(2):459. [PubMed: 10936141] 
50. MacIntyre NR, Cook DJ, Ely EW Jr, et al. Evidence-based guidelines for weaning and discontinuing ventilatory support: a collective task force facilitated by the American College of Chest Physicians; the American Association for Respiratory Care; and the American College of Critical Care Medicine. Chest. 2001; 120(6 Suppl):375S-395S. [PubMed: 11742959]

51. Blackwood B, Alderdice F, Burns KEA, Cardwell CR, Lavery G, O'Halloran P. Use of weaning protocols for reducing duration of mechanical ventilation in critically ill adult patients: Cochrane systematic review and meta-analysis. BMJ British medical journal (Clinical research ed ). 2011 Jan 13.342(2):c7237.

52. Morris PE, Goad A, Thompson C, et al. Early intensive care unit mobility therapy in the treatment of acute respiratory failure. Critical Care Medicine. 2008; 36(8):2238-2243. [PubMed: 18596631]

53. Schweickert WD, Pohlman MC, Pohlman AS, et al. Early physical and occupational therapy in mechanically ventilated, critically ill patients: a randomised controlled trial. Lancet. 2009; 373(9678):1874-1882. [PubMed: 19446324]

54. Needham DM, Korupolu R. Rehabilitation quality improvement in an intensive care unit setting: implementation of a quality improvement model. Top Stroke Rehabil. 2010; 17(4):271-281. [PubMed: 20826415]

55. Morrison RS, Penrod JD, Cassel JB, et al. Cost savings associated with US hospital palliative care consultation programs. Arch Intern Med. 2008; 168(16):1783-1790. [PubMed: 18779466]

56. Penrod JD, Deb P, Dellenbaugh C, et al. Hospital-Based Palliative Care Consultation: Effects on Hospital Cost. Journal of palliative medicine. 2010; 13(8):973-979. [PubMed: 20642361]

57. Norton SA, Hogan LA, Holloway RG, Temkin-Greener H, Buckley MJ, Quill TE. Proactive palliative care in the medical intensive care unit: Effects on length of stay for selected high-risk patients. Critical Care Medicine. 2007; 35(6):1530-1535. [PubMed: 17452930]

58. Garland A, Shaman Z, Baron J, Connors AF Jr. Physician-attributable differences in intensive care unit costs: a single-center study. Am J Respir Crit Care Med. 2006; 174(11):1206-1210. [PubMed: 16973977]

59. Scales DC, Laupacis A. Health technology assessment in critical care. Intensive care medicine. 2007; 33(12):2183-2191. [PubMed: 17952404]

60. Harvey S, Singer M. Managing critically ill patients with a pulmonary artery catheter. Br J Hosp Med (Lond). 2006; 67(8):421-426. [PubMed: 16918104]

61. Wiener RS, Welch HG. Trends in the use of the pulmonary artery catheter in the United States, 1993-2004. JAMA. 2007; 298(4):423-429. [PubMed: 17652296]

62. Pronovost P, Needham D, Berenholtz S, et al. An intervention to decrease catheter- related bloodstream infections in the ICU. N Engl J Med. 2006; 355(26):2725-2732. [PubMed: 17192537]

63. Weiss CH, Moazed F, McEvoy CA, et al. Prompting Physicians to Address a Daily Checklist and Process of Care and Clinical Outcomes: A Single-Site Study. Am J Respir Crit Care Med. 2011 201101-0037OC.

64. Girard TD, Kress JP, Fuchs BD, et al. Efficacy and safety of a paired sedation and ventilator weaning protocol for mechanically ventilated patients in intensive care (Awakening and Breathing Controlled trial): a randomised controlled trial. Lancet. 2008; 371(9607):126-134. [PubMed: 18191684]

65. Haynes AB, Weiser TG, Berry WR, et al. A Surgical Safety Checklist to Reduce Morbidity and Mortality in a Global Population. N Engl J Med. 2009; 360(5):491-499. [PubMed: 19144931]

66. Dellinger RP, Carlet JM, Masur H, et al. Surviving Sepsis Campaign guidelines for management of severe sepsis and septic shock. Crit Care Med. 2004; 32(3):858-873. [PubMed: 15090974]

67. Logan AC, Yank V, Stafford RS. Off-Label Use of Recombinant Factor VIIa in U.S. Hospitals: Analysis of Hospital Records. Annals of Internal Medicine. 2011; 154(8):516-522. [PubMed: 21502649]

68. Yank V, Tuohy CV, Logan AC, et al. Systematic Review: Benefits and Harms of InHospital Use of Recombinant Factor VIIa for Off-Label Indications. Annals of Internal Medicine. 2011; 154(8): 529-540. [PubMed: 21502651] 
69. Merlani P, Garnerin P, Diby M, Ferring M, Ricou B. Quality improvement report: Linking guideline to regular feedback to increase appropriate requests for clinical tests: blood gas analysis in intensive care. BMJ. 2001; 323(7313):620-624. [PubMed: 11557715]

70. Zimmerman JE, Alzola C, Von Rueden KT. The use of benchmarking to identify top performing critical care units: a preliminary assessment of their policies and practices. J Crit Care. 2003; 18(2):76-86. [PubMed: 12800117]

71. McAlpine L, Cohen IL, Truckenbrod A. Reducing resource consumption through work redesign in a surgical intensive care unit: A multidisciplinary, protocol-based progressive care area. Heart \& Lung: The Journal of Acute and Critical Care. 2007; 26(4):329-334.

72. Krieger BP, Ershowsky P, Spivack D. One Year's Experience With a Noninvasively Monitored Intermediate Care Unit for Pulmonary Patients. JAMA. 1990; 264(9):1143-1146. [PubMed: 2384938]

73. Gracey DR, Hardy DC, Koenig GE. The chronic ventilator-dependent unit: a lower- cost alternative to intensive care. Mayo Clinic proceedings. 2000; 75(5):445-449. [PubMed: 10807071]

74. Dasgupta A, Rice R, Mascha E, Litaker D, Stoller JK. Four-Year Experience With a Unit for Long-term Ventilation (Respiratory Special Care Unit) at the Cleveland Clinic Foundation. Chest. 1999; 116(2):447-455. [PubMed: 10453875]

75. Elpern E, Silver MR, Rosen RL, Bone RC. The noninvasive respiratory care unit. Patterns of use and financial implications. Chest. 1991; 99(1):205-208. [PubMed: 1898646]

76. Kahn JM, Benson NM, Appleby D, Carson SS, Iwashyna TJ. Long-term acute care hospital utilization after critical illness. JAMA. 2010; 303(22):2253-2259. [PubMed: 20530778]

77. Kahn JM, Goss CH, Heagerty PJ, Kramer AA, O'Brien CR, Rubenfeld GD. Hospital volume and the outcomes of mechanical ventilation. N Engl J Med. 2006; 355(1):41-50. [PubMed: 16822995]

78. Nathens AB, Jurkovich GJ, Maier RV, et al. Relationship Between Trauma Center Volume and Outcomes. JAMA: the journal of the American Medical Association. 2001; 285(9):1164-1171. [PubMed: 11231745]

79. Phibbs CS, Bronstein JM, Buxton E, Phibbs RH. The effects of patient volume and level of care at the hospital of birth on neonatal mortality. JAMA: the journal of the American Medical Association. 1996; 276(13):1054-1059. [PubMed: 8847767]

80. Hutchings A, Durand MA, Grieve R, et al. Evaluation of modernisation of adult critical care services in England: time series and cost effectiveness analysis. BMJ. 2009; 339(v11_2):b4353. [PubMed: 19906740]

81. Iwashyna TJ, Christie JD, Moody J, Kahn JM, Asch DA. The structure of critical care transfer networks. Med Care. 2009; 47(7):787-793. [PubMed: 19536030]

82. Kahn JM, Asch RJ, Iwashyna TJ, et al. Physician attitudes toward regionalization of adult critical care: a national survey. Crit Care Med. 2009; 37(7):2149-2154. [PubMed: 19455025]

83. Kahn JM, Linde-Zwirble WT, Wunsch H, et al. Potential value of regionalized intensive care for mechanically ventilated medical patients. Am J Respir Crit Care Med. 2008; 177(3):285-291. [PubMed: 18006884]

84. Cummings J, Krsek C, Vermoch K, Matuszewski K. Intensive care unit telemedicine: review and consensus recommendations. Am J Med Qual. 2007; 22(4):239-250. [PubMed: 17656728]

85. Thomas EJ, Lucke JF, Wueste L, Weavind L, Patel B. Association of telemedicine for remote monitoring of intensive care patients with mortality, complications, and length of stay. JAMA. 2009; 302(24):2671-2678. [PubMed: 20040555]

86. Morrison JL, Cai Q, Davis N, et al. Clinical and economic outcomes of the electronic intensive care unit: results from two community hospitals. Crit Care Med. 2010; 38(1):2-8. [PubMed: 19730249]

87. Lilly CM, Cody S, Zhao H, et al. Hospital Mortality, Length of Stay, and Preventable Complications Among Critically Ill Patients Before and After Tele-ICU Reengineering of Critical Care Processes. JAMA. 2011; 305(21):2175-2183. [PubMed: 21576622]

88. Franzini L, Sail KR, Thomas EJ, Wueste L. Costs and cost-effectiveness of a telemedicine intensive care unit program in 6 intensive care units in a large health care system. Journal of Critical Care. 2011; 26(3):329. [PubMed: 21376515] 
89. Scales DC, Dainty K, Hales B, et al. A multifaceted intervention for quality improvement in a network of intensive care units: a cluster randomized trial. JAMA. 2011; 305(4):363-372. [PubMed: 21248161] 


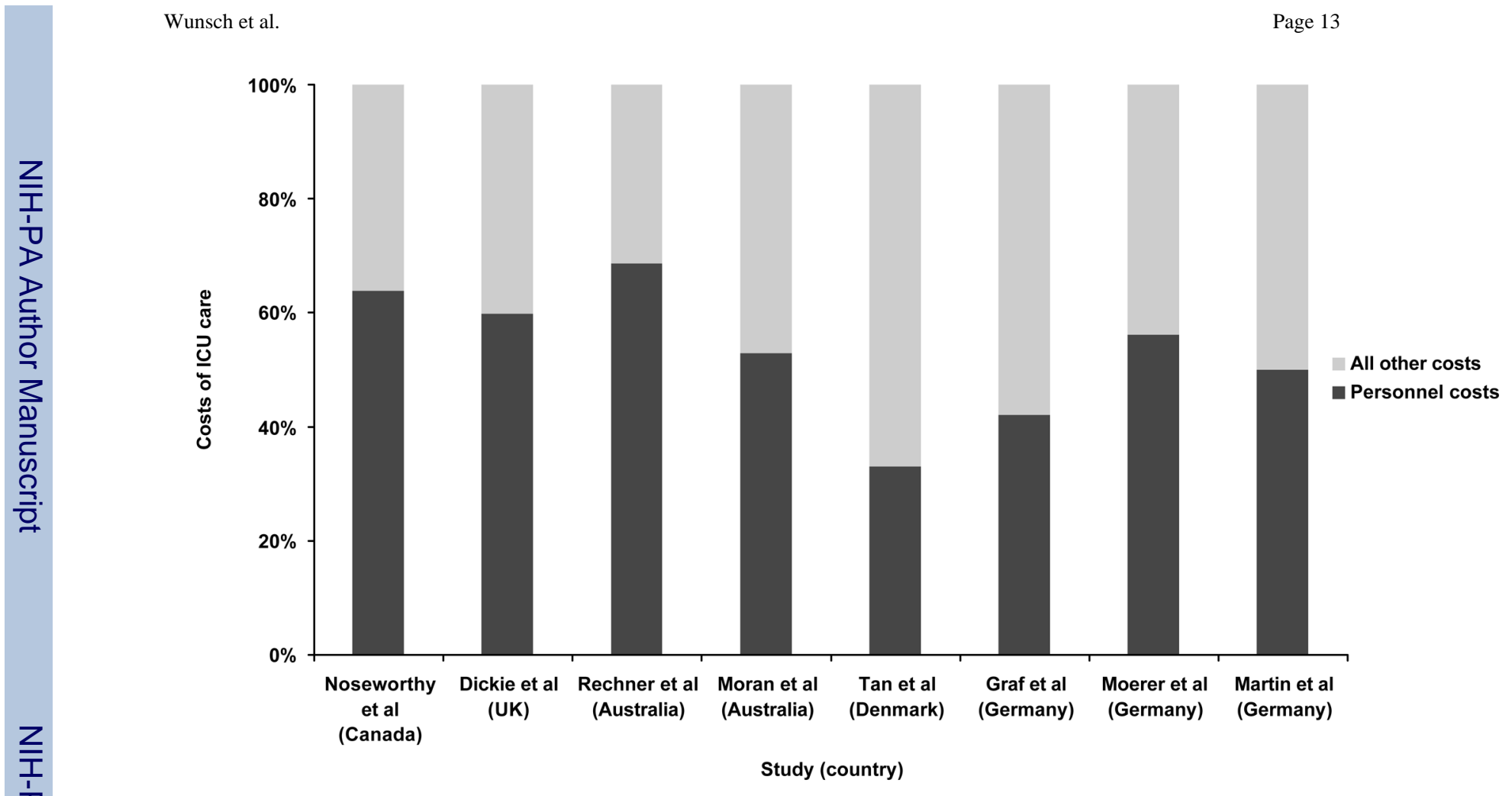

Figure 1.

Estimates of personnel costs associated with the ICU [13-20] 


\section{Table 1}

Examples of potential effects on hospital costs of different changes to ICU organization and management

\begin{tabular}{lccc}
\hline Organizational change & Possible clinical outcome & Fixed costs & Variable costs \\
\hline Closing ICU beds & Unclear & Decreased & Decreased \\
Intensivist staffing & Decreased mortality & Increased & Unclear \\
Pharmacist staffing & Fewer adverse drug events, deceased LOS & Increased & Decreased \\
Lower nurse to patient ratio & Fewer adverse events, decreased LOS & Increased & Unclear \\
Checklist prompter & Decreased mortality, decreased LOS & Increased & Decreased \\
\hline
\end{tabular}

